\title{
Simulation and Evaluation of a Simple Adaptive Antenna Array for a WCDMA Mobile Communication
}

\author{
Idigo V.E, Ifeagwu E.N,Azubogu A.C.O, Akpado K.A., Oguejiofor O.S \\ Department of Electronics and Computer Engineering, Nnamdi Azikiwe University Awka
}

\begin{abstract}
This paper presents a uniform Linear Array model of a simple adaptive antenna array based on signal-to-interference and noise ratio (SINR) maximization. The SINR using the adaptive antenna array was investigated for a conventional narrowband beam former by varying the number of antenna array elements and number of interfering signals or users. The results obtained were compared with that of omni-directional antenna. The graph obtained from the results showed significant improvement in SINR as the number of antenna elements increases in the presence of large interferers for odd numbered array.
\end{abstract}

Keywords-Smartantenna; bandwidth; SINR; adaptive beam forming.

\section{INTRODUCTION}

The Smart antennas technology is one of the leading innovations for achieving highly efficient networks that maximize network capacity and improve quality of service and coverage. Smart Antennas are arrays of antenna elements that change their antenna pattern dynamically to adjust to the noise, interference in the channel and mitigate multipath fading effects on the signal of interest[1]. In other words, smart antenna has a pattern that is not fixed but adapts to current radio conditions , and nulls out the interferers. Smart antenna systems are classified into three levels of intelligence on the basis of their transmit strategy [2,3].

Smart antennas provide greater capacity and performance benefits than omni-directional antennas because they can be used to customize and fine-tune antenna coverage pattern to the changing traffic or radio frequency (RF) conditions in a wireless communication system like the WCDMA network.

Beam forming (BF) in smart antenna technology is a process in which each user's signals is multiplied by complex weight vectors that adjust the magnitude and phase of the signal from each antenna element [4]. The beam forming appropriately combines the signals received by different elements of an antenna array to form a single output[5]. Many adaptive algorithms have been developed to determine the optimal weight vectors of antenna array elements dynamically, based on different performance criteria.

The weight vectors produce the desired radiation pattern that can be changed dynamically.

\section{BLOCK DIAGRAM AND IMPLEMENTATION.}

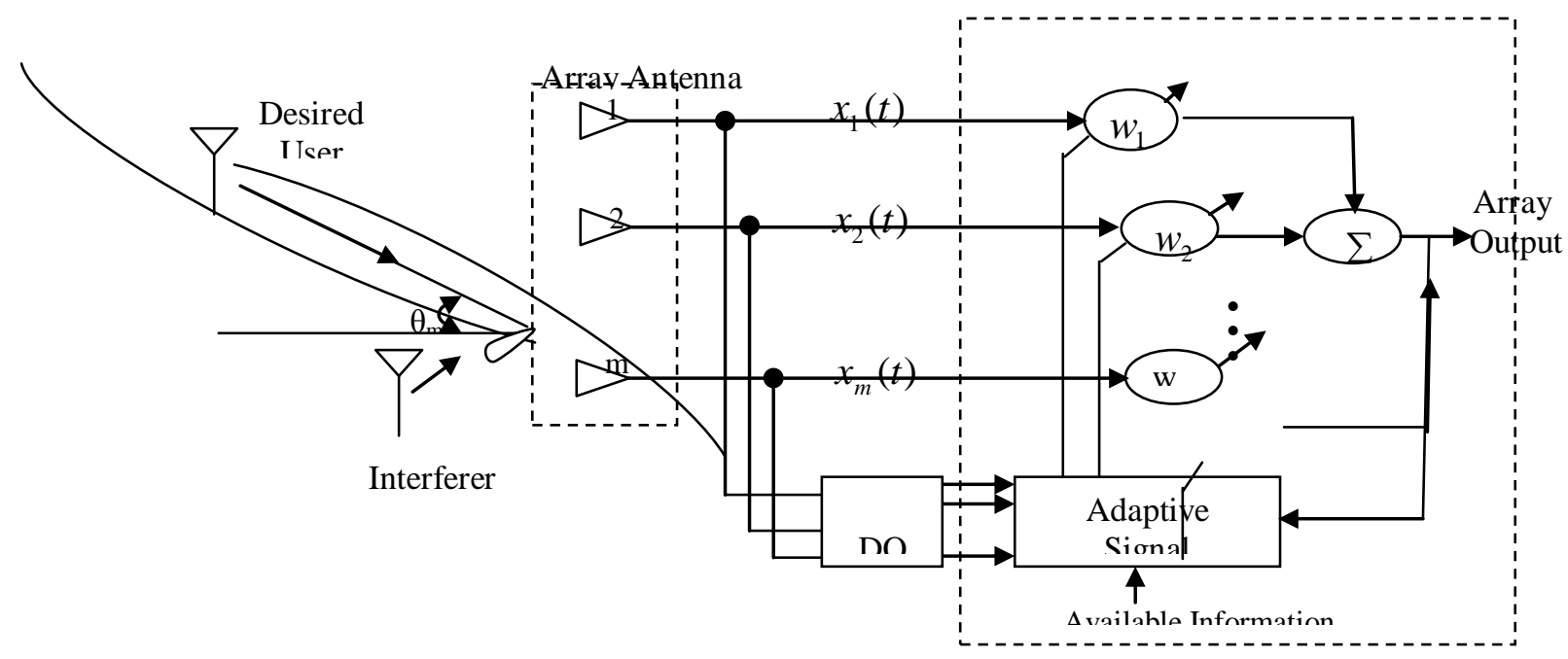

Beam forming

Figure 1: Functional Block Diagram of an Adaptive Antenna System [6] 
The block diagram of a simple adaptive antenna array is shown in Fig.1.The signals induced on an antenna array are multiplied by the adjustable complex weights

$\left(\mathrm{w}_{1}, \mathrm{w}_{2} \ldots \ldots \ldots \ldots \mathrm{w}_{\mathrm{m}}\right)$ and then combined to form the system output using adaptive beam forming algorithm[7].

\section{A. Mean path loss}

The path loss is used to describe the attenuation of radio signal as it travels in space[8]. The path loss model to be considered here is that of an outdoor environment.

When there are no obstacles between the BS and MS, the propagation characteristics are subject to free space propagation.

In this case, the path loss is given by :

$$
\operatorname{LP}_{\mathrm{f}}(\mathrm{dB})=32.44+20 \log _{10} \mathrm{f}_{\mathrm{c}}+20 \log _{10} \mathrm{~d}
$$

Where $\mathrm{LP}_{\mathrm{f}}$ is the free space path loss, fc is the carriers' frequency in $(\mathrm{MHz}), \mathrm{d}$ is the distance between the BS and MS in $(\mathrm{Km})$.

In the presence of obstacles the path loss model for urban and suburban environment are as follows [9]

$L P(d B)=69.55+26.16 \log _{10} f_{c}+\left(44.9-6.55 \log _{10} h_{b}\right)+\log d-$ $13.82 \operatorname{logh} \mathrm{h}_{\mathrm{b}}-\square(\mathrm{hm})$

Where $\square(\mathrm{hm})$ is the correlation factor for MS antenna height, $h_{b}$ is the height of BS $(\mathrm{km})$.

The correlation factor $\square\left(h_{m}\right)$ for MS antenna height is evaluated as

1) for large cities.

$\square\left(\mathrm{h}_{\mathrm{m}}\right)=8.29\left[\log _{10}(1.54 \mathrm{hm})\right]^{2}-1.1$,

$$
\begin{array}{r}
\mathrm{f}_{\mathrm{c}} \leq 200 \mathrm{MHz} \\
=3.2[\log 10(11.57 \mathrm{hm})] 2-4.97, \\
\mathrm{f}_{\mathrm{c}} \geq 400 \mathrm{MHz}
\end{array}
$$

Where $h_{m}$ is the height of MS.

2) For small and medium size cities

$\square(\mathrm{hm})=\left[1.1 \log _{10} \mathrm{f}_{\mathrm{c}}-0.7\right] \mathrm{h}_{\mathrm{m}}-[1.56 \operatorname{logfc}-0.8]$

For suburban area:

$\mathrm{Lp}_{\mathrm{s}}=\mathrm{Lp}-2\left[\log _{10}(\mathrm{fc} / 28)\right]^{2}-5.4$

For rural area:

$\mathrm{Lp} 0=\mathrm{Lp}-4.78\left(\log _{10} \mathrm{f}_{\mathrm{c}}\right)^{2}+18.33 \log _{10} \mathrm{f}_{\mathrm{c}}-40.94[\mathrm{~dB}]$

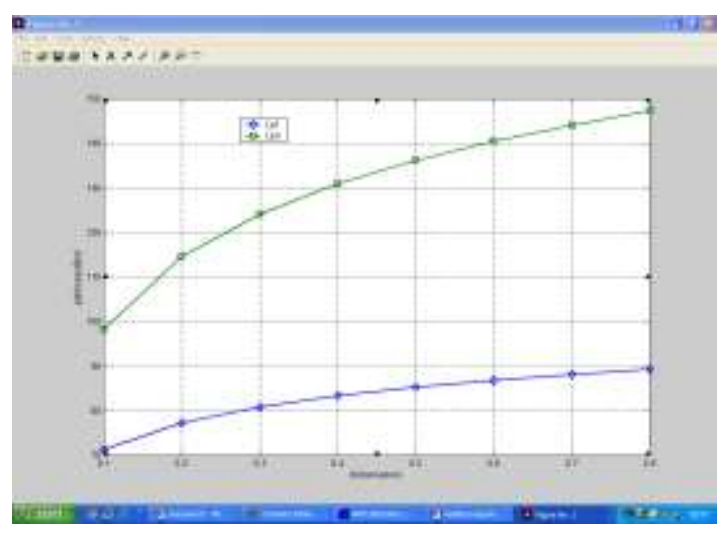

Figure 2. Path loss vs distance for a typical suburban area (with BS opposite B-division police station, awka).

The path loss in figure 2. is obtained for a suburban environment with the BS at B-division Police station opposite new state secretariat, Awka. The CDMA operator is Visafone Nigeria and the carrier frequency, $\mathrm{f}_{\mathrm{c}}$ was set at $878.1325 \mathrm{MHz}$ and reference power level is $-30 \mathrm{dBm}$. The height of the base station, $\mathrm{h}_{\mathrm{b}}$ is $120 \mathrm{ft}(0.03658 \mathrm{~km})$ and that of mobile station, $\mathrm{h}_{\mathrm{ms}}$ is taken to be $6 \mathrm{ft}(0.00183 \mathrm{~km})$.

To compute the path loss and the graph of figure 2 Matlab was used. The graph shows that path loss increases with distance. This path loss would be minimized or completely eliminated if adaptive antenna array is deployed at the BS.

\section{RESULTS AND ANALYSIS.}

A cell model deploying adaptive antenna array at BS is presented and received signal at the antenna elements is simulated and modeled by considering uniform linear array model. A real time measurement of signal strength, distance of MS from BS, and AOA of arriving signal from uplink is carried out at a test bed belonging to Visafone Nigeria which is a CDMA carrier situated at B-division police station, Awka.

To evaluate the path loss of a typical WCDMA environment we deployed sectorized antenna at the BS. The results obtained are helpful in the simulation and performance analysis of adaptive antenna array. All simulations are done in Matlab

\section{A. Strength and AOA measurement.}

The measurements were carried out using mobile monitoring system (MMS) equipment belonging to Nigerian Communication Commission, NCC. The signal strength is measured using spectrum analyzer and the AOA is seen on the visualizer. 
TABLE 1: SignAL StRENGTH (DBM) AND AOA $\left({ }^{0}\right)$ MEASUREMENTS.

\begin{tabular}{|c|c|c|}
\hline $\begin{array}{l}\text { Distance } \\
(\mathbf{m})\end{array}$ & $\begin{array}{l}\text { Signal strength } \\
(\mathbf{d B m})\end{array}$ & AOA $\left.\mathbf{(}^{\mathbf{0}}\right)$ \\
\hline 100 & -35.50 & 15 \\
\hline 200 & -46.29 & 20 \\
\hline 300 & -38.24 & 28 \\
\hline 400 & -30.03 & 30 \\
\hline 500 & 33.59 & 118 \\
\hline 600 & 42.67 & 105 \\
\hline 700 & -40.00 & 89 \\
\hline 800 & -40.17 & 74 \\
\hline
\end{tabular}

The measured results were obtained using base height (120ft),power level

$(-30 \mathrm{dBm})$, central frequency $(878.1325 \mathrm{~Hz})$.

TABLE 2: SINR WHEN THE NUMBER OF INTERFERERS IS TWO.

\begin{tabular}{|c|c|c|}
\hline$M$ & SINR $(\mathrm{d}=0.5 \lambda)$ & SINR $(\mathrm{d}=0.75 \lambda)$ \\
\hline 1 & 0.50 & 0.50 \\
\hline 2 & 0.10 & 0.45 \\
\hline 3 & 159.89 & 177.05 \\
\hline 4 & 9.97 & 838.13 \\
\hline 5 & 526.11 & 1899.80 \\
\hline 6 & 43.81 & 2310.70 \\
\hline 7 & 1103.30 & 1293.50 \\
\hline 8 & 102.54 & 160.06 \\
\hline 9 & 1549.70 & 1012.30 \\
\hline 10 & 125.66 & 1167.40 \\
\hline 11 & 1421.30 & 764.61 \\
\hline 12 & 63.86 & 100.57 \\
\hline
\end{tabular}

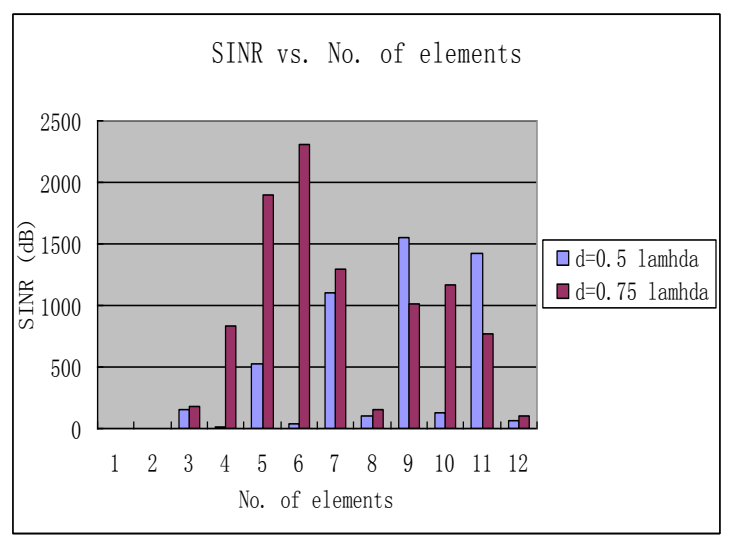

Figure 2. SINR vs. No. of antenna elements in the presence of two interferers.

Figure 2. shows that there is significant improvement in the SINR as the inter-element spacing increases between 1-element and 6-element array. Better performance is achieved in real time with inter-element spacing of $0.5 \lambda$ as seen in figure 2 when $\mathrm{M}=5,7,9$ or 11 .

TABLE 3: SINR OF OMNI-DIRECTIONAL ANTENNA AND 2-ELEMENT ARRAY.

\begin{tabular}{|c|c|c|}
\hline No. of interferers & $\begin{array}{c}\text { SINR for Omni- } \\
\text { directional }\end{array}$ & $\begin{array}{c}\text { SINR for } \\
\text { 2-array }\end{array}$ \\
\hline 2 & 0.50 & 0.10 \\
\hline 4 & 0.25 & 0.60 \\
\hline 6 & 0.17 & 0.81 \\
\hline 8 & 0.13 & 0.88 \\
\hline 10 & 0.10 & 0.92 \\
\hline 12 & 0.08 & 0.94 \\
\hline
\end{tabular}

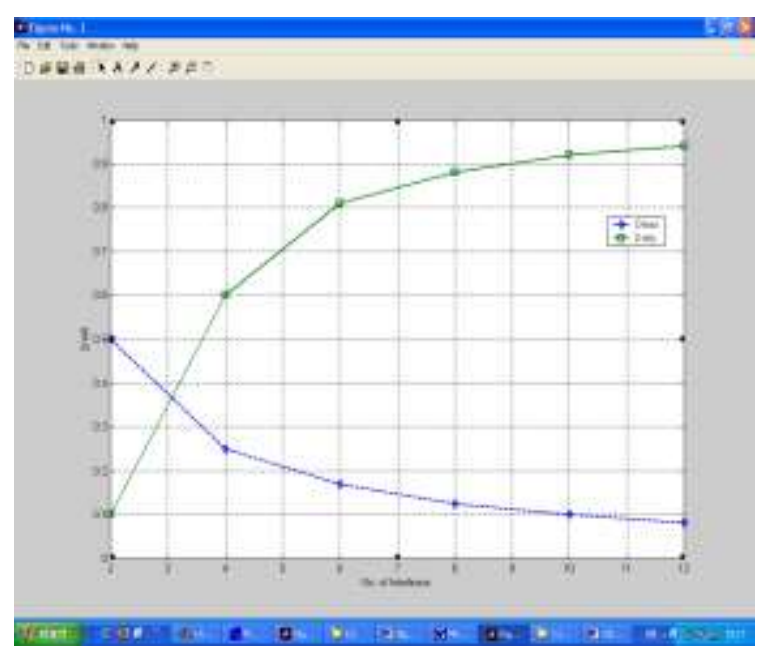

Fig. 3: SINR of Omni-directional antenna and 2-element array.

Figure 3. shows that adaptive antenna array increases the SINR as the number of interferers increases, while the omnidirectional decreases the SINR as the number of interferers increases..

\section{SUMMARY OF RESULTS.}

The graphs obtained for SINR vs. No. of antenna elements showed that better performance is achieved as the number of antenna elements and interferers increase with inter-element spacing of $0.5 \lambda$. The graphs for SINR against the number of interferers reveals that odd numbered arrays give significant performance improvement compared to their even numbered counterpart for our model.

Therefore, from the analysis, 9-ary or 11-ary would be the best choice for designers . Since our interest is in deploying a simple adaptive antenna at BS, 5-element and 7-element arrays are ideal for the proposed system as they also showed significant improvement in SINR over standard antennas. The graphs also reveal over 9 folds SINR for 2-ary against omnidirectional antenna system while higher arrays showed over 1500 folds in the presence of large interferers 


\section{CONCLUSION}

From the charts, it was observed that the signal-tointerference and noise ratio depends on the number of antenna element, the inter-element spacing between the arrays and the number of interferers.

There was great improvement in the SINR when odd numbered elements were used with inter-element spacing of $\mathrm{d}=0.5 \lambda$ in the presence of large interferers. Adaptive antenna as observed from our analysis showed greater improvement in the SINR over omni-directional antenna in the presence of large interferers.

Finally, improving the interference suppression and noise reduction capabilities of any antenna system in the presence of large interferers, increases the capacity of that system deploying such an antenna. Therefore, adaptive antenna proposed here will increase capacity of a WCDMA network.

\section{REFERENCES}

[1] A.Boukalov "Introduction to smart Antenna Technologies and
Algorithms", 2009, workshop on smart Antenna Technology and Application, RAWCON.

[2] A.F. Naguib "Adaptive Antenna for CDMA wireless Networks", 2008, Department of Electrical Engineering, Stanford University.

[3] S. R. Saunders, "Antennas and propagation for wireless communication systems", 2009, John Wiley and son.

[4] Bernard Widrow and Samuel D. Stearns, "Adaptive Signal Processing," 2008, Prentice Hall, New Jersey.

[5] J. Fuhl, A. Kuchar, and E. Bonek, "Capacity increase in cellular PCS by smart antennas", 2007, IEEE Transactions on Vehicular Technology, vol.3, pp. $1962-1966.0$

[6] R. Kawitkar, "Issues in deploying smart Antennas in Moblie Radio Networks," 2008, Proceeding World Academy of Science, Engineering And Technology, vol 31.

[7] Applebaum S. "Adaptive Arrays," 2008, IEEE Transactions on Antenna and propagation, vol 24, No 5, pp 585-598.

[8] Chris Loadman, Zhizhang chen, and Dylan Jorgensen, "An Overview of Adaptive Antenna Technologies for wireless communication", 2009, Communication Networks and service Research conference, New Brunwick, Canada.

[9] J.H Winters, "Smart Antenna Techniques and their application to wireless Ad-hoc networks", 2006, IEEE Trans. On Wireless communication, vol.13, pp 77-83. 